\title{
Diameter Tuning of Single-Walled Carbon Nanotubes by Diffusion Plasma CVD
}

\author{
Toshiaki Kato, Shunsuke Kuroda, and Rikizo Hatakeyama \\ Department of Electronic Engineering, Tohoku University, Sendai 980-8579, Japan \\ Correspondence should be addressed to Toshiaki Kato, kato12@ecei.tohoku.ac.jp \\ Received 22 June 2010; Accepted 16 August 2010 \\ Academic Editor: Jianyu Huang
}

Copyright () 2011 Toshiaki Kato et al. This is an open access article distributed under the Creative Commons Attribution License, which permits unrestricted use, distribution, and reproduction in any medium, provided the original work is properly cited.

We have realized a diameter tuning of single-walled carbon nanotubes (SWNTs) by adjusting process gas pressures with plasma chemical vapor deposition (CVD). Detailed photoluminescence measurements reveal that the diameter distribution of SWNTs clearly shifts to a large-diameter region with an increase in the pressure during plasma CVD, which is also confirmed by Raman scattering spectroscopy. Based on the systematical investigation, it is found that the main diameter of SWNTs is determined by the pressure during the heating in an atmosphere of hydrogen and the diameter distribution is narrowed by adjusting the pressure during the plasma generation. Our results could contribute to an application of SWNTs to high-performance thin-film transistors, which requires the diameter-controlled semiconductor-rich SWNTs.

\section{Introduction}

Single-walled carbon nanotubes (SWNTs) [1] have attracted intense attention due to their prominent electrical and optical properties [2-6]. One of the most promising electrical applications of SWNTs is to fabricate a thin-film field-effect transistor (FET) exploiting their flexible structure and high carrier mobility [7-9]. Since the mixture of metallic SWNTs in the FET channel increases leak currents between source and drain electrodes, which results in low on/off ratios, the selective growth of semiconducting SWNTs is urgently required. The band gap of SWNTs and contact resistance between nanotubes and electrodes are strongly influenced by the tube diameter $[10,11]$. Thus, it is indispensable to grow diameter-controlled semiconductor-rich SWNTs for realizing high-performance SWNTs-based thin-film FETs. It is known that the SWNTs grown by plasma chemical vapor deposition (CVD) show a tendency to contain semiconducting SWNTs with concentration higher than that by the other growth methods such as arc discharge, laser ablation, and thermal CVD, while their detailed mechanisms are still under investigation [12-15]. Although plasma CVD can be one of the promising approaches to obtain the abovementioned well-diameter-controlled semiconductor-rich SWNTs, the difficulty in controlling the plasma conditions has gotten in the way of realizing the precise diameter tuning of SWNTs.

Here, we demonstrate the diameter tuning of SWNTs by changing the gas pressure during the CVD process. Detailed photoluminescence (PL) and Raman scattering spectroscopy analyses reveal that the main diameter of SWNTs becomes large with an increase in the gas pressure. The systematic investigation is also carried out to figure out the mechanism of the diameter variation.

\section{Experimental}

2.1. SWNTs Growth. A home-made diffusion plasma CVD system was utilized for the growth of freestanding SWNTs $[16,17]$. The Fe (thickness $(t): 0.5 \sim 1 \mathrm{~nm}) / \mathrm{Al}_{2} \mathrm{O}_{3}(t: 20 \mathrm{~nm})$ multilayer catalyst was formed on an Ag substrate $(t$ : $0.2 \mathrm{~mm}$ ) by a vacuum evaporation and a sputtering method, respectively. A capacitively coupled plasma was generated by supplying a radio-frequency (RF: $13.56 \mathrm{MHz}$ ) power $\left(P_{\mathrm{RF}}\right)$ to an upper electrode. A mesh grid was used as an anode to promote spatial diffusion of plasmas. A substrate was placed on a heater which was located underneath the lower anode-electrode. The distance between the lower electrode and the substrate was fixed at $70 \mathrm{~mm}$. The growth 


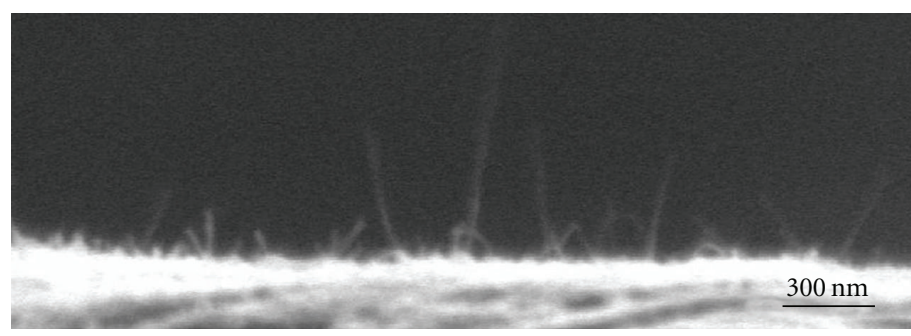

(a)

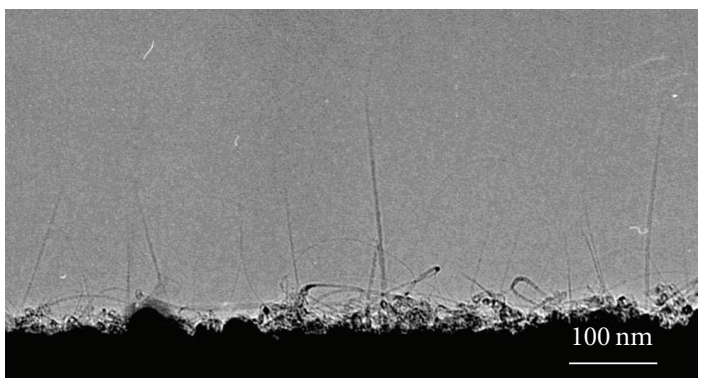

(b)

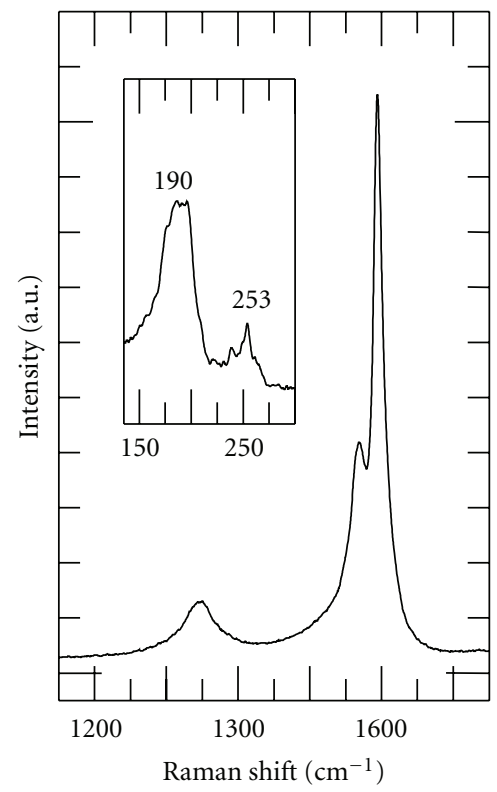

(d)

Figure 1: (a) SEM and (b), (c) TEM images of freestanding individual SWNTs. (d) Raman scattering spectrum (488 nm excitation) of freestanding individual SWNTs. Inset of $(\mathrm{d})$ is emphasis of the RBM region.

of SWNTs by plasma CVD was carried out with the following procedures. First, the system was pumped down to a base pressure of $10^{-2} \mathrm{~Pa}$ with rotary and diffusion pumps. The substrate was heated up to $600^{\circ} \mathrm{C}$ under an Ar flow, and then the Ar gas was immediately switched to a methane and hydrogen mixture gas (3:7 mixture ratio). Note that a total pressure during the heating process was set to be as the same as that during the growth unless otherwise specified. When the total pressure reached a desired pressure (20-650 Pa), the $P_{\mathrm{RF}}$ of $80 \mathrm{~W}$ was supplied to generate plasmas and the SWNT growth was started. The growth time was $20 \mathrm{sec}$. After the SWNT growth, the methane and hydrogen gases were pumped out and an Ar gas was introduced into the system in order to cool down the substrate.

2.2. Electron Microscope Analysis. As-grown states of freestanding SWNTs were analyzed with a scanning electron microscope (SEM, Hitachi 4100) and a transmission electron microscope (TEM, Hitachi HF-2000). A thin Cu wire (dia. = $100 \mu \mathrm{m}$ ) covered by the $\mathrm{Fe} / \mathrm{Al}_{2} \mathrm{O}_{3}$ catalyst was utilized as a substrate, which was able to be directly set in a TEM holder without any conventional TEM grid.

2.3. Photoluminescence Analysis. Photoluminescence-excitation (PLE) measurements were performed with a JY (Horiba) Fluorolog-3 system. The excitation wavelength was varied from 500 to $828 \mathrm{~nm}$ in $4 \mathrm{~nm}$ step, and emission signals were accumulated for $20 \mathrm{sec}$ in each excitation step. Excitation and emission slit widths were fixed at $10 \mathrm{~nm}$. In order to cut the Rayleigh scattering, optical filters (Sigmakoki, ITF-50S-83IR, ITF-50S-85IR) were set in front of a liquid-nitrogen-cooled InGaAs array detector.
2.4. Raman Scattering Spectroscopy Analysis. Raman scattering spectra were taken with $488 \mathrm{~nm}$ Ar laser and $632.8 \mathrm{~nm}$ He-Ne laser excitations. As-growth SWNTs were used for this Raman scattering analysis.

\section{Results and Discussion}

Figures 1(a)-1(c) show typical SEM (a) and TEM (b, c) images of the as-grown SWNTs produced by diffusion plasma CVD. SWNTs are found to be grown in the freestanding form [17]. The existence of SWNTs is also confirmed by a Raman scattering spectrum as shown in Figure 1(d). In general, electrostatic potentials in plasmas sharply drop in the interface between the plasmas and solid materials, forming strong electric fields on the surface of the solid materials. Since the polarization constant of SWNTs in an axial direction is extremely high, the strong dipole moment is induced in the axial tube direction in the presence of electric fields. Due to the energy stability of the dipole moments under the electric fields, SWNTs tend to align with the electric fields. This is a possible explanation for the freestanding growth of SWNTs in the case of plasma CVD [18].

PL spectroscopy is a powerful tool to assign each chirality of semiconducting SWNTs [19, 20]. Since excited excitons in the semiconducting SWNTs are easily quenched through the metallic SWNTs, each SWNT has to be well isolated to observe the occurrence of the optical emission. Thus, PL measurements of SWNTs are usually carried out with the dispersed SWNTs in the specific chemical solution. Owing to the unique freestanding as-grown state of SWNTs grown by diffusion plasma CVD, it is possible to obtain the PL signal 


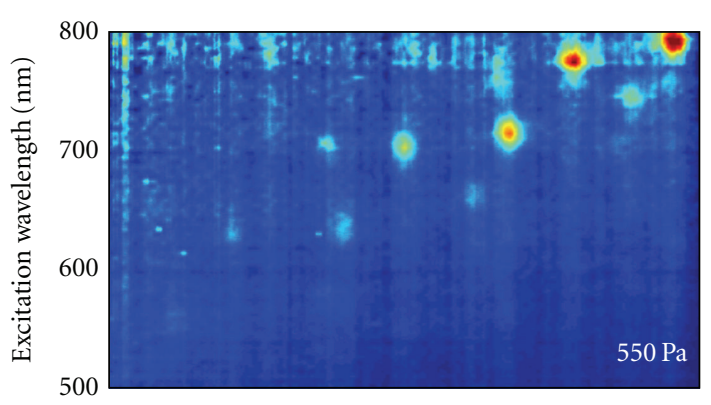

(a)

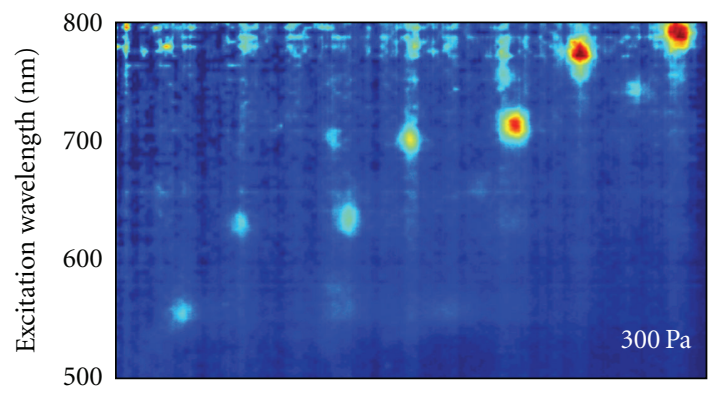

(b)

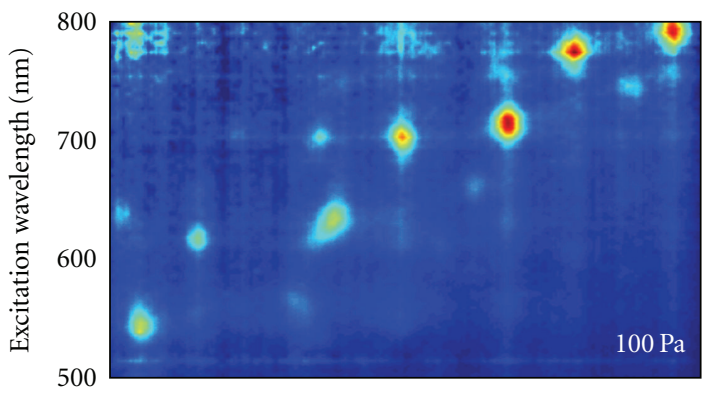

(c)

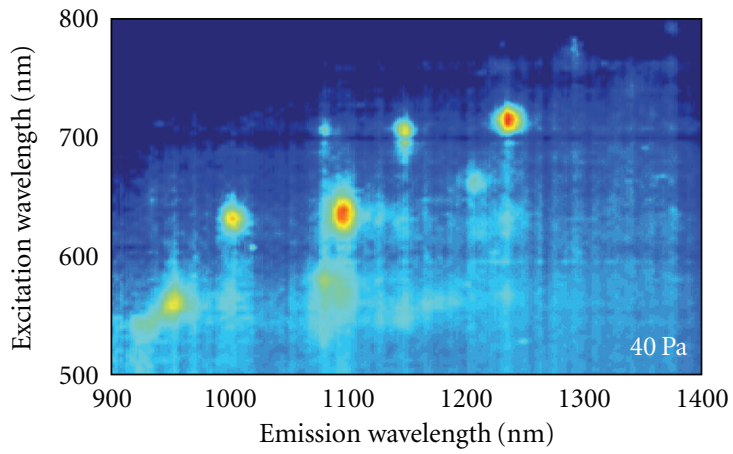

(d)
Diameter $(\mathrm{nm})$

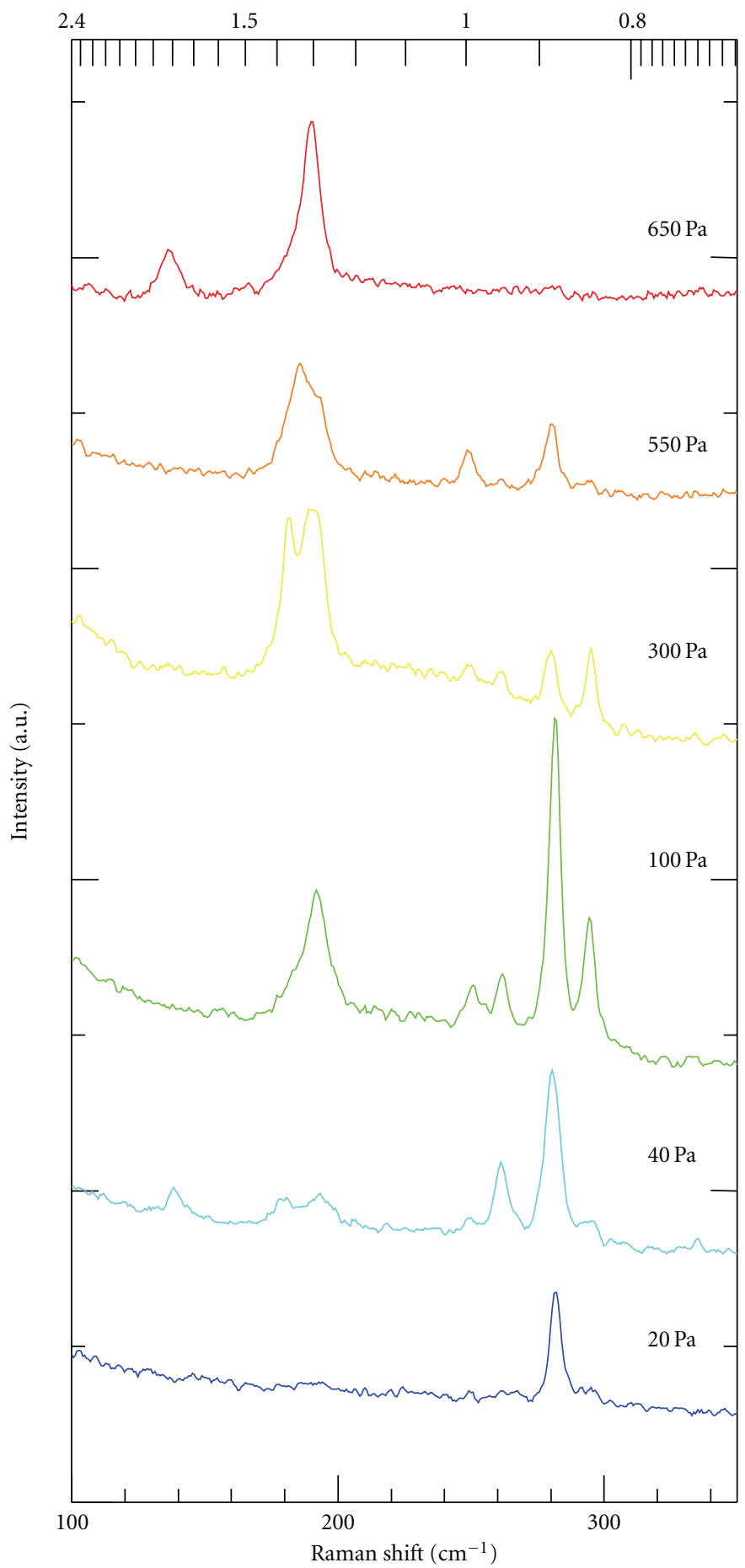

(e)

Figure 2: (a-d) PLE maps of as-grown freestanding SWNTs grown at the different pressures: (a) $550 \mathrm{~Pa}$, (b) $300 \mathrm{~Pa}$, (c) $100 \mathrm{~Pa}$, and (d) $40 \mathrm{~Pa}$, respectively. (e) Growth pressure dependence of RBM in Raman scattering spectra (632.8 nm excitation) of as-grown freestanding SWNTs.

from the as-grown SWNTs without any chemical dispersion [21].

Figures 2(a)-2(d) give PLE maps of as-grown SWNTs produced at different gas pressures. It is to be noted that all the PLE measurements are carried out immediately after the growth process in order to prevent the freestanding SWNTs from forming bundles, which causes significant PL changes [21]. It is found that peaks in the PLE map at the high growth pressure (Figure 2(a)) tend to appear in the range of long excitation and emission wavelengths. The peak 


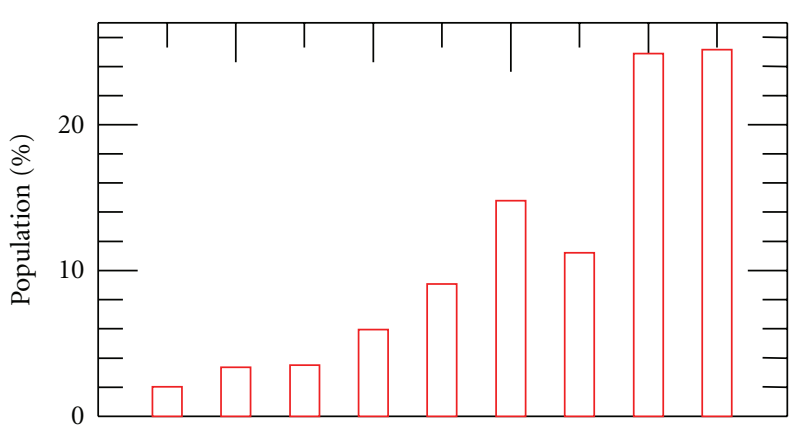

(a)

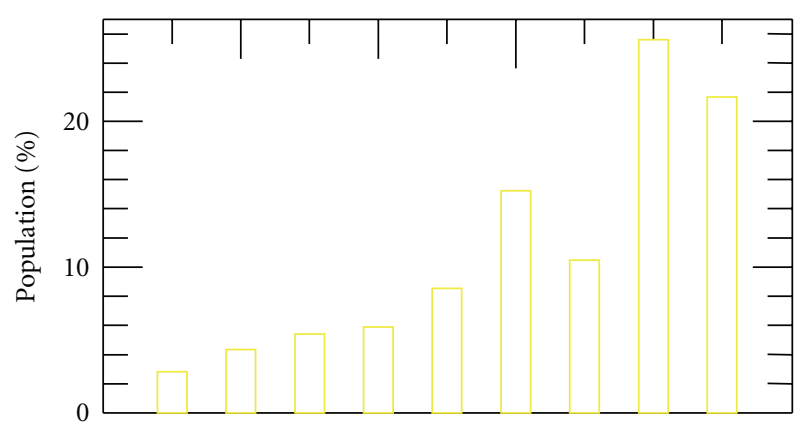

(b)

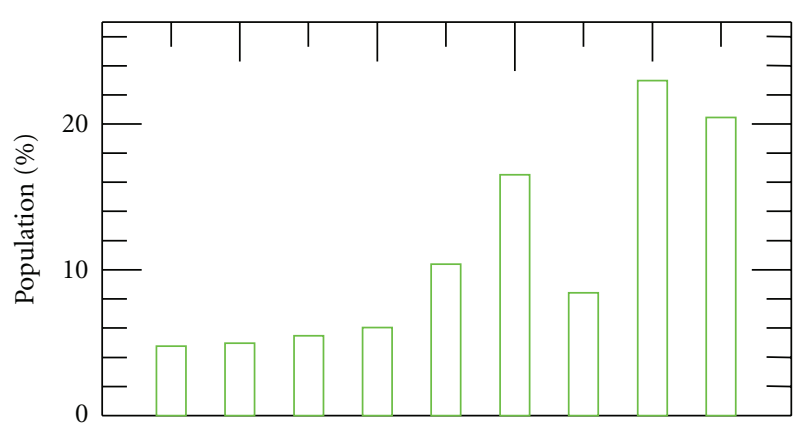

(c)

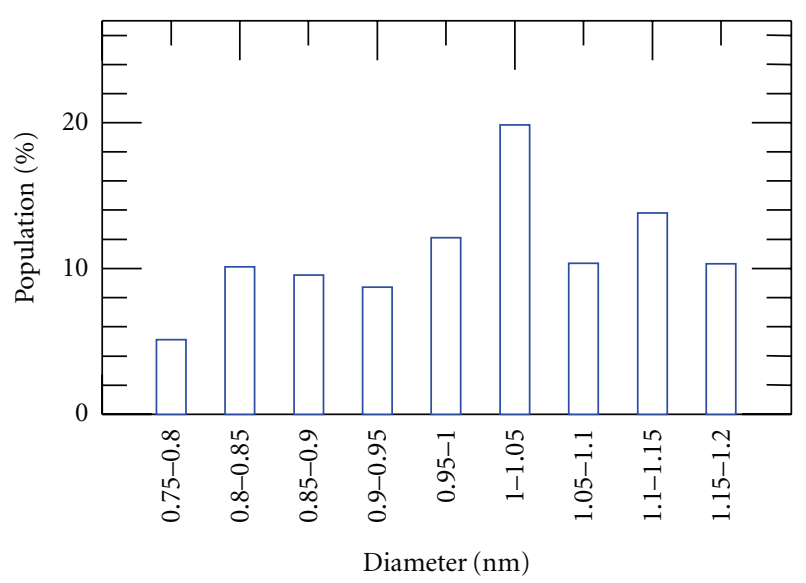

(d)

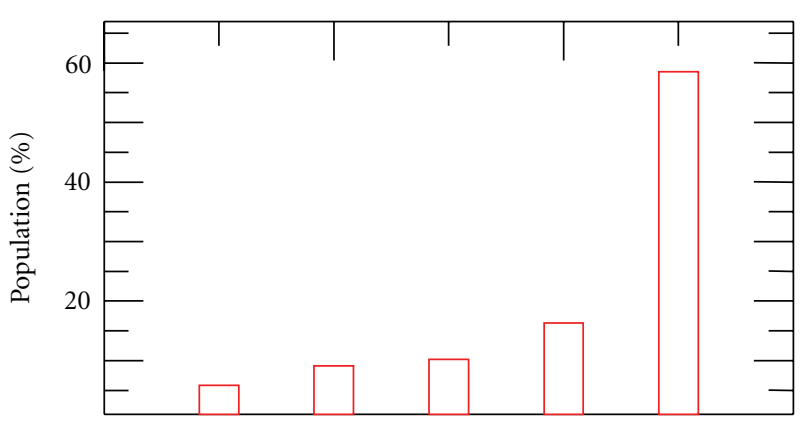

(e)

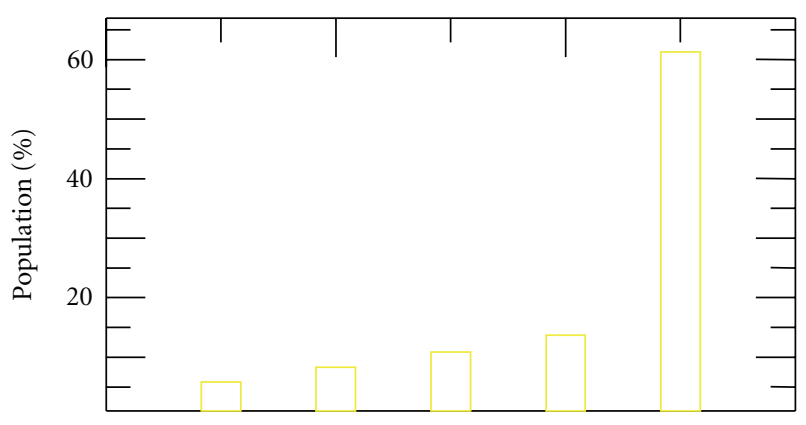

(f)

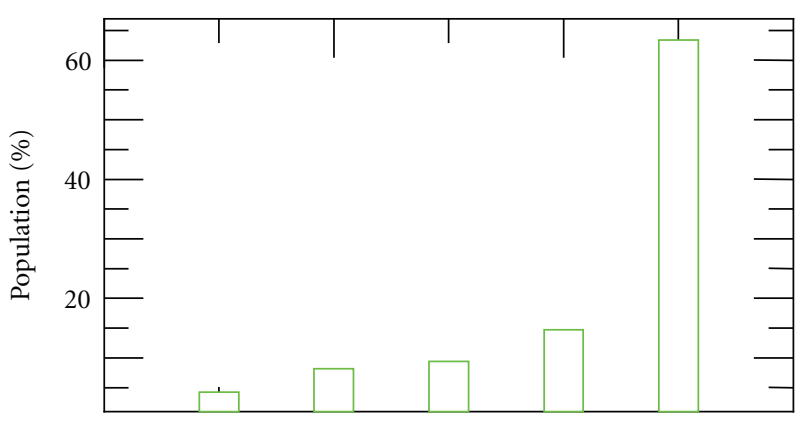

(g)

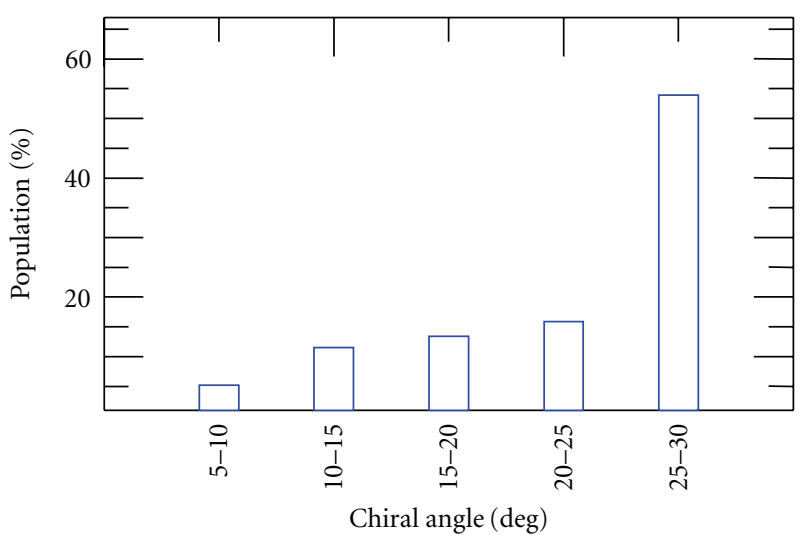

(h)

FIgURE 3: Population histograms as functions of $(\mathrm{a}-\mathrm{d})$ SWNTs diameters and (e-h) chiral angles, which are grown at the different pressures: (a, e) $550 \mathrm{~Pa},(\mathrm{~b}, \mathrm{f}) 300 \mathrm{~Pa},(\mathrm{c}, \mathrm{g}) 100 \mathrm{~Pa}$, and (d, h) $40 \mathrm{~Pa}$, respectively. 


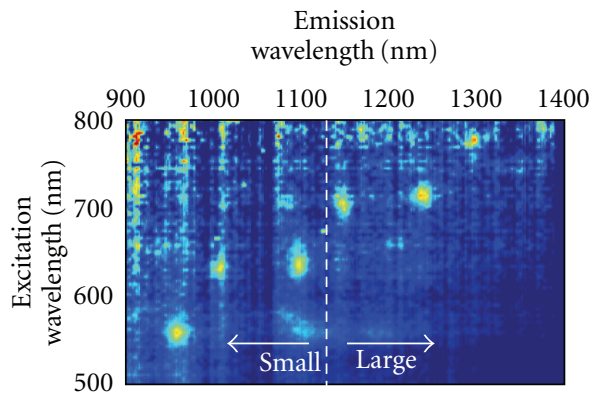

(a)

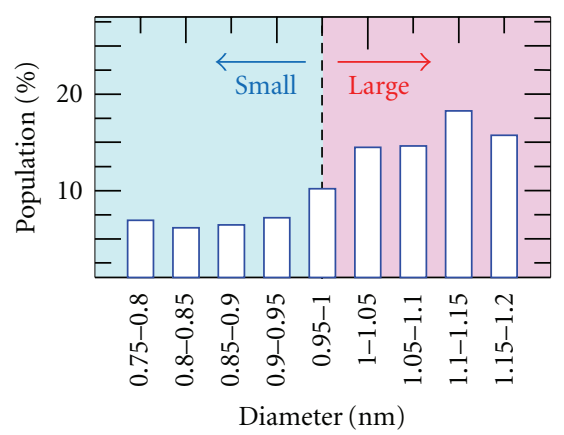

(d)

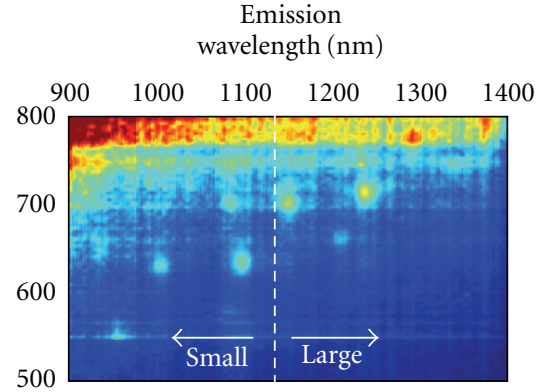

(b)

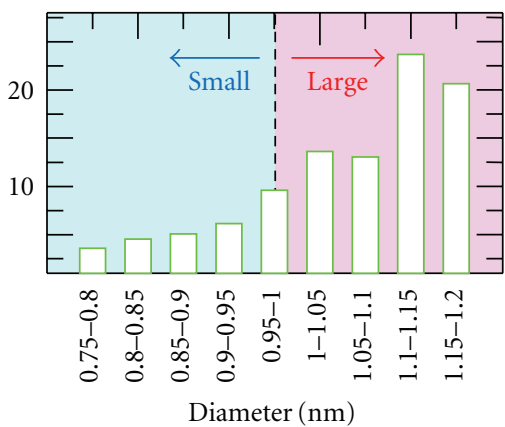

(e)

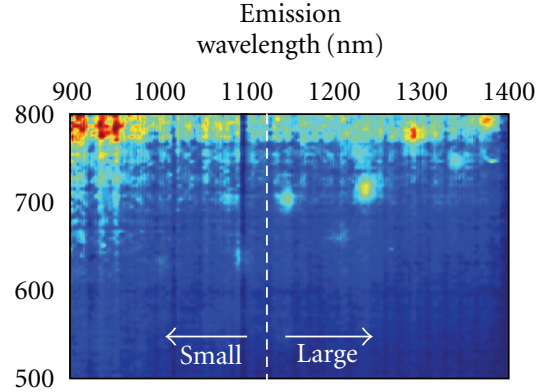

(c)

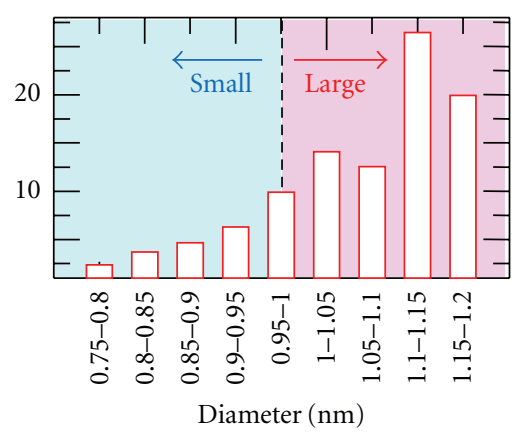

(f)

FIgURE 4: $(\mathrm{a}-\mathrm{c})$ PLE maps and $(\mathrm{d}-\mathrm{f})$ population-diameter histograms of as-grown freestanding SWNTs. The pressures during heating and growth are (a, d) both $60 \mathrm{~Pa},(\mathrm{~b}, \mathrm{e}) 500 \mathrm{~Pa}$ and $60 \mathrm{~Pa}$, and (c, f) both $500 \mathrm{~Pa}$, respectively.

positions shift to the region of short excitation and emission wavelengths with a decrease in the growth pressure (Figures $2(b)-2(d))$. Since each peak corresponds to each chirality in the sample and smaller-diameter SWNTs are positioned in the shorter wavelength region, the peak-position shift in the PLE map indicates that the diameter distribution of produced SWNTs is strongly influenced by the growth pressure. Thus, lower pressure enables the SWNTs diameter to become smaller. This diameter dependence on the growth pressure is also reflected in Raman scattering spectra of SWNTs grown at the different growth pressures. Figure 2(e) demonstrates that peak positions of the radial breathing mode (RBM) clearly shift from higher to lower values of wave number with an increase in the growth pressures. Here the RBM peak position and the SWNTs diameter are known to have a close correlation, which is $\omega=248 / d$ [22], where $\omega$ and $d$ are RBM peak position $\left(\mathrm{cm}^{-1}\right)$ and diameter $(\mathrm{nm})$, respectively. This result is fairly consistent with the PLE result shown in Figures 2 (a) $-2(d)$. The typical pressure range where SWNTs can be grown is from $30 \mathrm{~Pa}$ to $650 \mathrm{~Pa}$, which depends on the $P_{\mathrm{RF}}$ used for the plasma generation. Although the absolute intensity of G-band in Raman scattering spectra decreases in the low or high pressure range, the G-band to D-band ratio is almost the same. This indicates that the quality of SWNTs should be the same in any pressure range whereas the density of SWNTs depends on the pressure. When we increase the input $P_{\mathrm{RF}}$, it is possible to grow SWNTs even below $30 \mathrm{~Pa}$, which means that the lack of hydrocarbon supply is significant under the lower pressure condition, and hence additional input $P_{\mathrm{RF}}$ is required to increase the density of active species used for the growth of SWNTs.

Since the optical absorption and emission efficiency of each SWNT depend on its chirality, the PL emission intensity does not directly correspond to the population of each chirality SWNT. To discuss the population of SWNTs, it is therefore required to inspect the optical absorption and emission efficiency of each chirality SWNT. Figures $3(a)-3(d)$ and $3(\mathrm{e})-3(\mathrm{~h})$ are SWNTs population histograms as the functions of diameter and chiral angle of SWNTs produced at the different growth pressures. These data are calculated from the absolute PL intensities shown in Figures 2(a)-2(d) and the optical efficiencies obtained from the theoretical calculation [23]. It is worthy of being noted that the detectable diameter range is $0.75-1.2 \mathrm{~nm}$ in this study due to the limitation of the PL detector used. The larger diameters of SWNTs (1.1$1.2 \mathrm{~nm}$ ) are confirmed to be dominant in the samples grown at the high pressures, and the main diameter shifts to around $1.0 \mathrm{~nm}$ with a decrease in the growth pressure. In contrast, there is no clear difference of chiral angle-among the growth pressures. In all the cases, near-armchair (25-30 deg) SWNTs show the highest population, which is similar to the SWNTs grown by the other methods [24]. The mechanism of the near-armchair rich growth is still unclear. In general, the chirality can be determined by the initial cap structure formed 
on the catalyst particle surface. For the stable cap formation, it is required to satisfy the isolated pentagon rule [25]. This cap structure stability might have effects on the higher population of near-armchair SWNTs than the other types of SWNTs.

In order to understand the mechanism of diameter shift depending on the process pressure, we carried out the systematic investigation. Since the pressures during the heating and growth are the same in our growth process, the process pressure affects both the heating and growth process. In order to clarify which exercises a critical effect on the diameter tuning of SWNTs, we performed experiments on the SWNTs growth under the following conditions; the pressures during the heating and growth are (1) both $60 \mathrm{~Pa}$, (2) $500 \mathrm{~Pa}$ and $60 \mathrm{~Pa}$, and (3) both $500 \mathrm{~Pa}$, respectively. Figures $4(\mathrm{a})-4(\mathrm{c})$ and $4(\mathrm{~d})-4(\mathrm{f})$ are PLE maps and population-diameter histograms of SWNTs produced under the conditions (1) ( $a$ and d), (2) (b and e), and (3) (c and f), respectively. For the simplification, we define the diameter ranges $0.75-1.0 \mathrm{~nm}$ and $1.0-1.2 \mathrm{~nm}$ as "small" and "large," respectively. When we compare the conditions (1) and (2), it is found that the population of small-diameter SWNTs decreases but the population of large-diameter SWNTs increases. Although we do not have any evidence about the catalyst particle size change after the high-pressure annealing, we believe that the catalyst size becomes large due to their aggregation after the highpressure annealing, which results in the growth of largerdiameter SWNTs because the other growth conditions are completely the same for these two samples. Interestingly, the clear difference is also found between the conditions (2) and (3). Although the population of large-diameter SWNTs is almost the same, the growth of small-diameter SWNTs is obviously suppressed. This can be explained as follows. The density of reactive hydrocarbon radials and ions should increase under the higher growth-pressure conditions. In the high carbon-supply condition, the small catalyst can be deactivated due to the oversupply of hydrocarbons, and hence the population of small-diameter SWNTs decreases. These indicate that the heating pressure is important to control the catalyst size distribution, which directly influences the main diameter of SWNTs, and that the pressure during plasma CVD is also important to narrow the SWNTs diameter distribution.

\section{Conclusions}

We have investigated the diameter distributions of plasma CVD-grown SWNTs in their as-grown state by PLE mapping and Raman scattering spectroscopy analyses. The pressure dependence study reveals that the process pressures strongly influence the SWNTs diameter distribution in plasma CVD. Furthermore, it is found that the catalyst particle size distribution can determine the main diameter of SWNTs and that their distribution can be narrowed by adjusting the plasma conditions. Our results could contribute to precisely and perfectly controlling the structure of as-grown SWNTs in the near future.

\section{References}

[1] S. Iijima and T. Ichihashi, "Single-shell carbon nanotubes of 1nm diameter," Nature, vol. 363, no. 6430, pp. 603-605, 1993.

[2] S. J. Tans, M. H. Devoret, H. Dai et al., "Individual singlewall carbon nanotubes as quantum wires," Nature, vol. 386, no. 6624, pp. 474-477, 1997.

[3] S. J. Tans, A. R. M. Verschueren, and C. Dekker, "Roomtemperature transistor based on a single carbon nanotube," Nature, vol. 393, no. 6680, pp. 49-52, 1998.

[4] A. Bachtold, P. Hadley, T. Nakanishi, and C. Dekker, "Logic circuits with carbon nanotube transistors," Science, vol. 294, no. 5545, pp. 1317-1320, 2001.

[5] A. Javey, J. Guo, Q. Wang, M. Lundstrom, and H. Dai, "Ballistic carbon nanotube field-effect transistors," Nature, vol. 424, no. 6949, pp. 654-657, 2003.

[6] M. Freitag, J. C. Tsang, J. Kirtley et al., "Electrically excited, localized infrared emission from single carbon nanotubes," Nano Letters, vol. 6, no. 7, pp. 1425-1433, 2006.

[7] Z. Wu, Z. Chen, X. Du et al., "Transparent, conductive carbon nanotube films," Science, vol. 305, no. 5688, pp. 1273-1276, 2004.

[8] M. C. LeMieux, M. Roberts, S. Barman, Y. W. Jin, J. M. Kim, and Z. Bao, "Self-sorted, aligned nanotube networks for thinfilm transistors," Science, vol. 321, no. 5885, pp. 101-104, 2008.

[9] M. Engel, J. P. Small, M. Steiner et al., "Thin film nanotube transistors based on self-assembled, aligned, semiconducting carbon nanotube arrays," ACS Nano, vol. 2, no. 12, pp. 24452452, 2008.

[10] R. Saito, G. Dresselhaus, and M. S. Dresselhaus, Physical Properties of Carbon Nanotubes, Imperial College Press, London, UK, 1998.

[11] W. Kim, A. Javey, R. Tu, J. Cao, Q. Wang, and H. Dai, "Electrical contacts to carbon nanotubes down to $1 \mathrm{~nm}$ in diameter," Applied Physics Letters, vol. 87, no. 17, Article ID 173101, 3 pages, 2005.

[12] Y. Li, D. Mann, M. Rolandi et al., "Preferential growth of semiconducting single-walled carbon nanotubes by a plasma enhanced CVD method," Nano Letters, vol. 4, no. 2, pp. 317321, 2004.

[13] L. Qu, F. Du, and L. Dai, "Preferential syntheses of semiconducting vertically aligned single-walled carbon nanotubes for direct use in FETs," Nano Letters, vol. 8, no. 9, pp. 2682-2687, 2008.

[14] U. J. Kim, E. H. Lee, J. M. Kim, Y.-S. Min, E. Kim, and W. Park, "Thin film transistors using preferentially grown semiconducting single-walled carbon nanotube networks by water-assisted plasma-enhanced chemical vapor deposition," Nanotechnology, vol. 20, no. 29, Article ID 295201, 2009.

[15] T. Mizutani, H. Ohnaka, Y. Okigawa, S. Kishimoto, and Y. Ohno, "A study of preferential growth of carbon nanotubes with semiconducting behavior grown by plasma-enhanced chemical vapor deposition," Journal of Applied Physics, vol. 106, no. 7, Article ID 073705, 5 pages, 2009.

[16] T. Kato, G.-H. Jeong, T. Hirata, R. Hatakeyama, K. Tohji, and K. Motomiya, "Single-walled carbon nanotubes produced by plasma-enhanced chemical vapor deposition," Chemical Physics Letters, vol. 381, no. 3-4, pp. 422-426, 2003.

[17] T. Kato, R. Hatakeyama, and K. Tohji, "Diffusion plasma chemical vapour deposition yielding freestanding individual single-walled carbon nanotubes on a silicon-based flat substrate," Nanotechnology, vol. 17, no. 9, pp. 2223-2226, 2006. 
[18] T. Kato and R. Hatakeyama, "Formation of freestanding single-walled carbon nanotubes by plasma-enhanced CVD," Chemical Vapor Deposition, vol. 12, no. 6, pp. 345-352, 2006.

[19] M. J. O'Connell, S. M. Bachilo, C. B. Huffman et al., "Band gap fluorescence from individual single-walled carbon nanotubes,” Science, vol. 297, no. 5581, pp. 593-596, 2002.

[20] S. M. Bachilo, M. S. Strano, C. Kittrell, R. H. Hauge, R. E. Smalley, and R. B. Weisman, "Structure-assigned optical spectra of single-walled carbon nanotubes," Science, vol. 298, no. 5602, pp. 2361-2366, 2002.

[21] T. Kato and R. Hatakeyama, "Exciton energy transfer-assisted photoluminescence brightening from freestanding singlewalled carbon nanotube bundles," Journal of the American Chemical Society, vol. 130, no. 25, pp. 8101-8107, 2008.

[22] A. Jorio, R. Saito, J. H. Hafner et al., "Structural (n, m) determination of isolated single-wall carbon nanotubes by resonant Raman scattering," Physical Review Letters, vol. 86, no. 6, pp. 1118-1121, 2001.

[23] Y. Oyama, R. Saito, K. Sato et al., "Photoluminescence intensity of single-wall carbon nanotubes," Carbon, vol. 44, no. 5, pp. 873-879, 2006.

[24] Y. Miyauchi, S. Chiashi, Y. Murakami, Y. Hayashida, and S. Maruyama, "Fluorescence spectroscopy of single-walled carbon nanotubes synthesized from alcohol," Chemical Physics Letters, vol. 387, no. 1-3, pp. 198-203, 2004.

[25] H. W. Kroto, "The stability of the fullerenes $\mathrm{C}_{\mathrm{n}}$, with $n=24$, 28, 32, 36, 50, 60 and 70," Nature, vol. 329, no. 6139, pp. 529$531,1987$. 

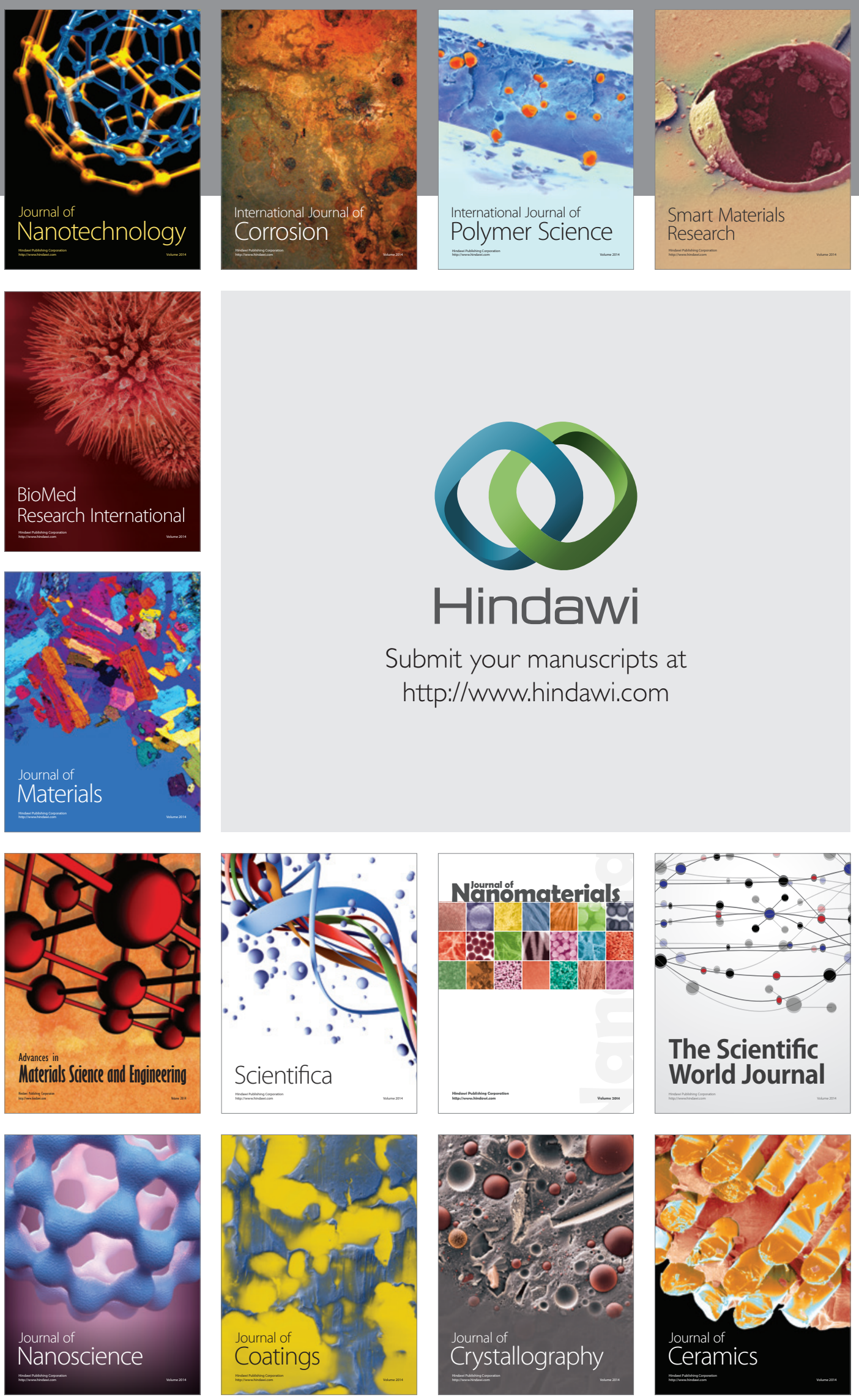

The Scientific World Journal

Submit your manuscripts at

http://www.hindawi.com

\section{World Journal}

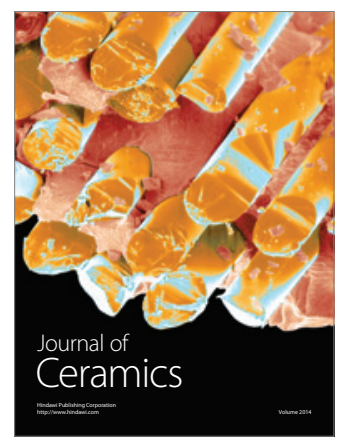

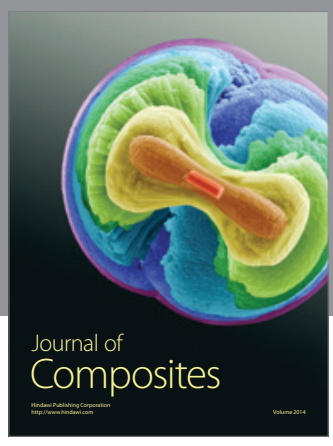
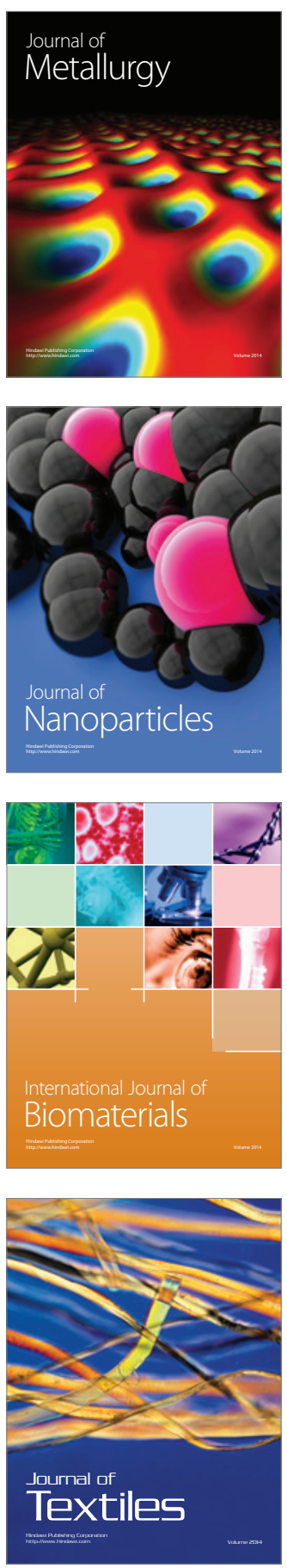